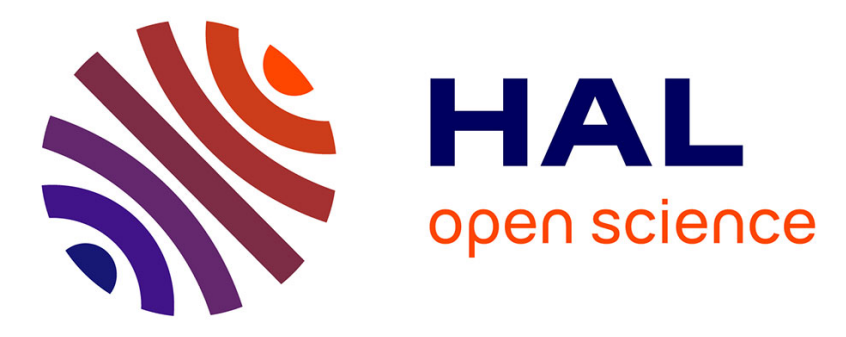

\title{
Graph-Search Descent and Approach Trajectory Optimization Based on Enhanced Aircraft Energy Management
}

Ramon Andreu Altava, Jean-Claude Mere, Daniel Delahaye, Thierry Miquel

\section{- To cite this version:}

Ramon Andreu Altava, Jean-Claude Mere, Daniel Delahaye, Thierry Miquel. Graph-Search Descent and Approach Trajectory Optimization Based on Enhanced Aircraft Energy Management. AIAA Aviation 2019 Forum, Jun 2019, Atlanta, United States. 10.2514/6.2019-3618 . hal-02170467

\section{HAL Id: hal-02170467 \\ https://hal-enac.archives-ouvertes.fr/hal-02170467}

Submitted on 12 Jul 2019

HAL is a multi-disciplinary open access archive for the deposit and dissemination of scientific research documents, whether they are published or not. The documents may come from teaching and research institutions in France or abroad, or from public or private research centers.
L'archive ouverte pluridisciplinaire HAL, est destinée au dépôt et à la diffusion de documents scientifiques de niveau recherche, publiés ou non, émanant des établissements d'enseignement et de recherche français ou étrangers, des laboratoires publics ou privés. 


\title{
Graph-Search Descent and Approach Trajectory Optimization Based on Enhanced Aircraft Energy Management
}

\author{
Ramon Andreu Altava* and Jean Claude Mere ${ }^{\dagger}$ \\ Airbus Operations SAS, Toulouse, 31300, France \\ Daniel Delahaye $\frac{\ddagger}{\ddagger}$ and Thierry Miquel ${ }^{\S}$ \\ ENAC, University of Toulouse, 31400, France
}

\begin{abstract}
ir Traffic growth requires a modernization of current Air Transportation System in A ment poses aviation development at risk. Under this context, trajectory optimization projects constitute a relevant mean to reduce fuel consumption and decrease the likelihood of go-around events as a result of a non-stabilized approach. The generation of a continuous optimal trajectory would provide flight crews with an useful tool to manage aircraft flight path or directly automate the trajectory management. This papers proposes a novel methodology based on a tailored version of $\mathrm{A}^{*}$ algorithm that generates upstream optimal trajectories with the certainty of terminating at current aircraft position, and solves energy errors during the approach phase through flap and airbrakes adjustments. Three use cases are presented: two concerning the calculation of the vertical profile for a complete arrival procedure and one regarding the computation of a trajectory that dissipates the excess of energy of an aircraft located close to the runway threshold. On one hand, results show fuel savings of $6 \%$ and $12 \%$ for the analyzed case studies accompanied by a reduction of $3 \%$ descent time, which are obtained due to enhanced energy management; on the other hand, during high-energy situations the function design prioritizes safety instead of fuel optimization, and proposes a flight strategy to stabilize the aircraft energy-state before landing.
\end{abstract}

\section{Nomenclature}

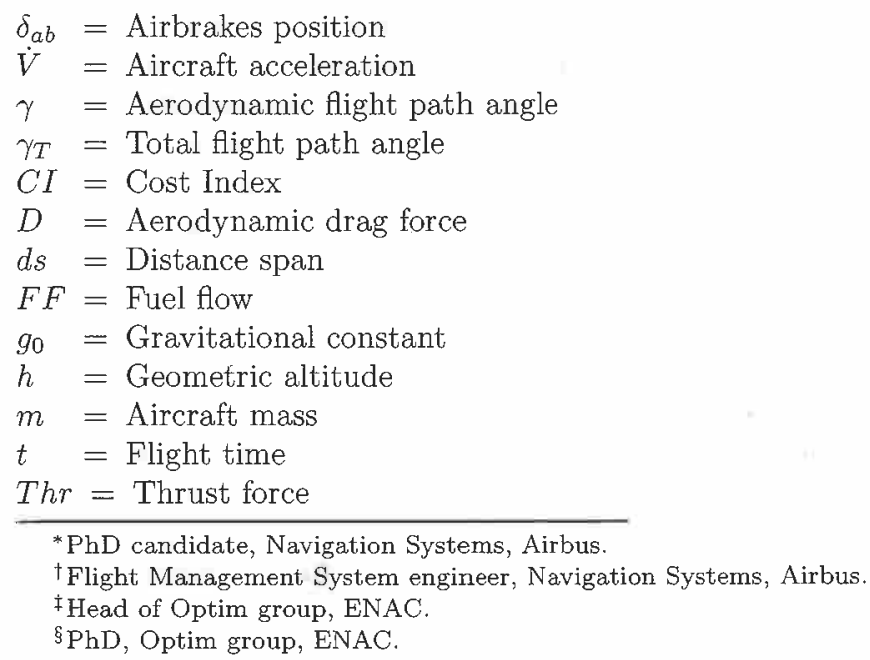


$V_{w}=$ Wind airspeed

$\mathrm{V}=$ True airspeed

\section{Introduction}

$\Lambda$ IR traffic is continuously increasing over the years ${ }^{1}$ and, in the more likely scenario, is predicted to double

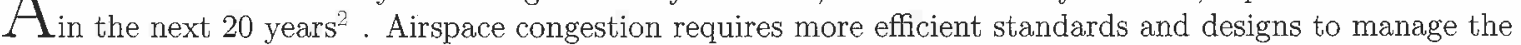
increasing number of operations ${ }^{3}$. Besides, current Air Transportation System (ATS) lacks of sustainability in the long-term and its impact on climate change is still one of the main challenges to cope with. The environmental footprint of aviation industry already constitutes a $3.6 \%$ of the total European greenhouse emissions ${ }^{4}$. Whilst most disrupting technologies are still on papers, several projects propose immediate actions and solutions to mitigate the environmental impact of aviation. From an airlines perspective, strong market competition makes that any fuel reduction is relevant for their business, since these savings rapidly escalate when considering the global picture of their operations and ultimately they can propose more competitive prices and operate new routes.

In recent years, flight efficiency has been largely improved through initiatives such as Free Route Airspace (FRA) $)^{5}$ or the introduction of Continuous Descent Operations (CDO) ${ }^{6}$, which have demonstrated to be generally more efficient than conventional step-down operations in terms of noise and gas emissions ${ }^{T}$. In major airports surrounded by large residential areas, airspace designers have introduced noise abatement procedures based on CDO that are operated during certain periods of time, usually in night time. The high workload and complex environment in descent and approach flight phases lead pilots to fail in managing optimally the aircraft trajectory, with the consequent impact on flight efficiency and, occasionally, on safety. This study aims at optimizing descent and approach phases based on CDO paradigm, and supporting pilots with energy management tasks in order to ensure the efficiency of the flight.

The Flight Management System (FMS) entered into service in the early $1980 \mathrm{~s}^{8}$ and reduced navigation workload so that flight crew was reduced from three to two people. The system performs relevant functions ${ }^{9}$ such as navigation, flight planning (both lateral and vertical), performance and transmission of guidance and display commands. Regarding descent and approach phases, state-of-the-art FMS compute a path based on several parameters like the Cost Index (CI), cruise flight level, estimated mass at arrival and pilot-entered arrival procedure. This path consists of a concatenation of idle and geometric segments, the latter being those generated when an altitude constraint restricts the construction of the (idle) path. In general, geometric segments require auto-thrust adjustments to maintain a speed target while the elevator guides the aircraft through the vertical path. In contrast, idle segments set auto-thrust to idle while the elevator maintains the target speed. The integration of the equations of motion is performed with a set of simplifying hypotheses, which are relatively independent of the aircraft maneuvering capabilities for a given arrival procedure. More specifically, decelerations are computed by a fixed Energy Share Factor (ESF) that distributes the available energy between altitude and speed, permitting the aircraft to descend and decelerate at the same time. It supports CDO where aircraft no longer decelerate to approach speed in a level-flight segment at glide-slope capture altitude but along the profile as they descend. The main limitation of this approach is that depending on the aircraft performance and the arrival procedure, deceleration to approach speed might be initiated above 7000 feet, which requires to anticipate flap extension and leads to long approach procedures that, in some cases, require more fuel than conventional step-down profiles. Hence, FMS hypotheses are in practice not sufficient to obtain optimal trajectories, so the design could be enhanced taking into account aircraft weight, performance and the arrival procedure as proposed in this paper.

A second enhancement of current design is related to the fact that these trajectories are rarely followed in real operations, since Air Traffic Control (ATC) provide radar vectors that assure aircraft separation and the management of the arrival sequence, temporarily deviating aircraft from their intended routes. In this situation, flight crews manage aircraft trajectory without a reference as the vertical profile is computed based on a given lateral path that is no longer valid, and it is their responsibility to stabilize the aircraft before landing. Aircraft energy state is defined as the sum of potential and kinetic energy, so high-energy occurs when the aircraft is too fast, too high or both, while low-energy state implies that the aircraft is low or below its target speed. In flight operations, the term energy management refers to the continuous transformation 
of energy that occurs due to the use of flight controls as the aircraft descends to the destination. In the absence of a reference trajectory, pilots are responsible for managing aircraft energy state through control devices such as thrust levers, airbrakes, landing gear and flap settings that modify the aircraft energy rate. Thus, the decision-making process depends exclusively on their experience and expertise, and may lead to non-optimal energy management or go-around procedures in case of an unstabilized approach ${ }^{10}$. In the presence of unexpected winds or certain ATC instructions this situation can be degraded, which justifies the fact that decision-making aid tools would help pilots to manage efficiently aircraft energy-state, reduce workload or even automate energy management tasks, yielding important fuel savings and reducing the number of non-stabilized approaches.

Trajectory optimization is an Optimal Control Problem (OCP $)^{11}$ that is generally solved through direct, indirect or dynamic programming methods ${ }^{12}$. The Time and Energy Management Operations (TEMO) ${ }^{13}$ concept proposes a pseudo-spectral algorithm to compute trajectories that reduce the number of energy corrections in the presence of a required time of arrival. Noise and pollutant emissions are reduced and compared with real flight data at Frankfurt airport ${ }^{14}$. Concerning indirect methods, they are implemented in a real-time algorithm whose results are compared with those of a $\mathrm{FMS}^{15}$ in wind conditions ${ }^{16}$. $\mathrm{A}^{*}$ algorithm has been successfully implemented ${ }^{171819}$ to compute optimal trajectories but they rely on simple heuristic functions that represent no advantage with respect to classic Dijkstra's algorithm. In general and independently of the solving method, works in literature divide the trajectory optimization problem in a. series of bounded segments, so they are not applicable to any procedure, and optimality is degraded due to the imposed boundary conditions. In order to obtain the global optimal trajectory for any arrival procedure, a tailored version of the well-known $\mathrm{A}^{*}$ algorithm ${ }^{20}$ for the fuel minimization problem is presented in this paper. The search space is defined by a graph that contains the whole set of aircraft states that are progressively generated by the application of a certain control value. The objective of the paper is twofold: on one hand, the algorithm proves that fuel savings can be obtained by applying enhanced energy management concepts to generate the vertical profile. On the other hand, the algorithm aims to propose an optimal flight strategy to pilots in order to arrive stabilized before landing no matter what the energy-state of the aircraft is, so the trajectory generator defines the optimal sequence for flap extensions and deploys airbrakes if necessary accounting for safety aspects.

The paper is divided as follows; the complete mathematical formulation of the problem is presented in section II. The algorithm functioning principles are depicted in section III. Then, several case studies are presented and analyzed in section IV; two related to pure vertical profile optimization and one applied to high-energy scenarios in approach. Finally, section $V$ draws the conclusion and defines the future work and perspectives.

\section{Mathematical Formulation}

\section{A. Optimization Problem}

In this study, the aircraft is represented as a point-mass model, which presents a sufficient level of representativeness with a relatively low level of complexity ${ }^{21}$. The optimization problem aims at minimizing the flight cost, which is defined as the sum of fuel consumption and time along the trajectory:

$$
J=\min \int_{s_{0}}^{s_{f}}\left(F F+\frac{C I}{60}\right) \frac{1}{V \cos \gamma+V_{w}} d s
$$

where $C I$ is expressed in $\frac{\$ / m i n}{\$ / k g}=\frac{k g}{m i n}$ and $F F$ in $\frac{k g}{s}$.

However, since fuel is of prime interest for airlines and additionally reduces the environmental footprint, this paper considers that $C I=0$, so the problem is restricted to the optimization of fuel expenditure. The FMS trajectory is computed through an Airbus Performance Database (PDB) that contains engines, aerodynamics and performance data in a set of look-up tables and loaded in the aircraft. The algorithm presented in this paper accommodates an Airbus FMS model through a genuine PDB, nevertheless, performance-related data is not displayed for confidentiality reasons. 


\section{B. Aircraft Model}

The Airbus FMS model integrated in the PDB contains an engine, aerodynamic and performance model of the given aircraft type.

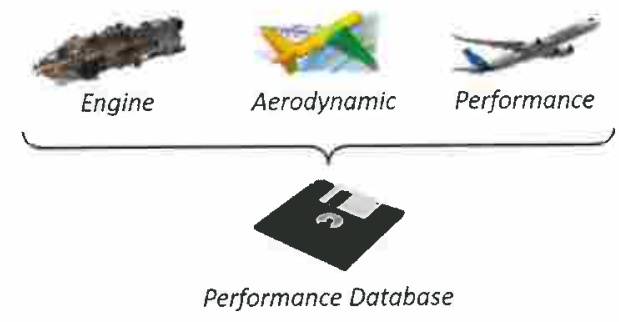

Figure 1. Models contained within a FMS Performance Database (PDB).

The engine model contains thrust maximum and minimum (idle) settings whose use depends on the flight phase. The Thrust Setting Parameter (TSP) is a scalable factor independent of the engine type, which is defined by:

$$
T S P_{\min , \max }=f(h, M)
$$

Thrust minimum and maximum ratings are computed by means of Eq. (2).

$$
T h r_{\min , \max }=f(T S P, M)
$$

In certain cases, thrust is computed by general equations of motion and TSP value is iterated from Eq. (3). Eventually, fuel flow is computed through the following expression:

$$
F F=f(T S P, h, M)
$$

Energy-state representations are usually a preferred manner to simplify the formulation of the equations of motion. Defining aircraft specific energy as the sum of potential and kinetic energy independent of aircraft mass, the derivation of the term with respect to time yields the energy rate, also known as energy height:

$$
\dot{E}_{T}=\dot{E}_{k}+\dot{E}_{p}=V \sin \gamma+\frac{V \dot{V}}{g_{0}}
$$

The Energy Share Factor (ESF) is defined as the percentage of total energy rate attributed to kinetic energy whilst the remaining goes to potential energy:

$$
E S F=\frac{\dot{E}_{k}}{\dot{E}_{k}+\dot{E}_{p}}
$$

The combination of Eqs. (5) and (6) lead to the following expression:

$$
\sin \gamma=\frac{(1-E S F)}{E S F} \frac{\dot{V}}{g_{0}}
$$

A further simplification is done through the introduction of the total flight path angle $\left(\gamma_{T}\right)$, which is defined as the sum of the aerodynamic flight path angle and the resulting acceleration or deceleration:

$$
\sin \gamma_{T}=\sin \gamma+\frac{\dot{V}}{g_{0}}=\frac{\sin \gamma}{1-E S F}=\frac{T h r-D\left(h, V, \delta_{a b}\right)}{m g_{0}}
$$

In this paper, the classic time-dependent equations of motion for a point-mass aircraft model are rewritten in terms of distance-dependent as procedure constraints are easily managed. The introduction of Eqs. (7) 
and (8) results in the following formulation:

$$
\left\{\begin{aligned}
h^{\prime} & =\frac{V(1-E S F) \gamma_{T}}{V \cos \gamma+V_{w}} \\
V^{\prime} & =\frac{g_{0} E S F \gamma_{T}}{V \cos \gamma+V_{w}} \\
m^{\prime} & =\frac{d m}{d s}=\frac{-F F}{V \cos \gamma+V_{w}}
\end{aligned}\right.
$$

As a result of the distance-dependent equations of motion presented in Eq. (9), the state vector is defined as $x(s)=[s, h, V, m, t$, Conf $]$, thereby $u(s)=\left[E S F, \gamma_{T}, \delta_{a b}\right]$ describes the control variables of the problem.

\section{Problem Constraints}

Constraints on state variables are dictated by the arrival procedures design and contained in an Airbus Navigation Database (NDB) ${ }^{22}$ under the form of altitude and speed constraints, the former imposing restrictions on aircraft altitude:

$$
\left\{\begin{array}{l}
\text { AT OR ABOVE } \rightarrow h_{\text {max }} \geq h \geq h_{C S T R} \\
\text { AT OR BELOW } \rightarrow h_{\text {min }} \leq h \leq h_{C S T R} \\
\text { AT } \rightarrow h=h_{C S T R}
\end{array}\right.
$$

Similarly, a speed constraint limits aircraft speed below a certain value:

$$
V_{C A S_{\text {min }}} \leq V_{C A S} \leq V_{C A S_{C S T R}}
$$

Furthermore, ATC regulation generally imposes a maximum speed of $250 \mathrm{kt}$ Calibrated Airspeed (CAS) for all aircraft below FL100:

$$
V_{C A S_{\min }} \leq V_{C A S} \leq V_{C A S_{S P D L I M}} \quad \forall h \leq F L 100
$$

For operational reasons, minimum speed is limited to $250 \mathrm{kt}$ when aircraft is above FL100. In addition to previous boundaries, aircraft speed is limited according to the flight envelope that is defined by the stall speed $\left(V_{L S}\right)$ and the maximum operating speed $\left(V_{M O}\right)$ :

$$
V_{L S} \leq V_{C A S} \leq V_{M O}
$$

During the approach phase, flap configuration is sequentially changed, resulting in an increase of the stall speed $\left(V_{L S}\right)$ and upper bounded by the maximum flap extended speed $\left(V_{F^{\prime} E}\right)$ :

$$
V_{L S} \leq V_{C A S} \leq V_{F E} \quad \forall \text { Conf } \neq \text { clean } \Longrightarrow \text { Conf } \in[\text { clean, } 1,2,3,4]
$$

In order to account for passenger comfort, longitudinal accelerations are limited by:

$$
\dot{V} \leq 0.07 g_{0}
$$

Control variables $\gamma_{T}$ and $E S F$ are bounded between a maximum and minimum value; thrust power limits the total flight path angle while ESF is operationally limited for passenger comfort purposes:

$$
\left.\gamma_{T_{\text {min }}}\right|_{T h r=T h r_{i d l e}} \leq \gamma_{T} \leq\left.\gamma_{T_{\min }}\right|_{T h r=T h r_{\max }}
$$

Climb segments $(\gamma>0)$ are forbidden per design so that any $\gamma_{T}>0$ corresponds to a speed acceleration.

$$
E S E \in[-0.5, \ldots, 1]
$$

A decelerated level-flight segment corresponds to a value of $E S F=1$, while $E S F<0$ leads to an acceleration as a result of a drastic energy potential loss. $E S F=0$ keeps constant true-airspeed as aircraft descends. Airbrakes extension impacts on the aerodynamic force and the deflection angle is limited between:

$$
0 \leq \delta_{A B} \leq \delta_{A B_{\max }}
$$




\section{Trajectory Optimization Algorithm}

The algorithm implemented for the computation of optimal trajectories is a version of $\mathrm{A}^{*}$ completely adapted and generalized ${ }^{23}$ to comply with any existing arrival procedure, and assure that the path reaches aircraft position. A* algorithm uses a heuristic function that estimates for each node the cost-to-go to attain the target node. This estimation shall be always lower (optimistic) than the actual cost to comply with admissibility criterion. Furthermore, a consistent heuristic function decreases along the path and permits to introduce a closed list that contains all the visited nodes, so the computation is generally faster but requires additional memory to store the nodes. In this paper, the heuristic function is an estimation of the optimal fuel consumption and is calculated considering the Manhattan distance from any node to the target multiplied by the fuel flow at target altitude, which represents the lowest value from all search space, and represents an optimistic estimation of the actual optimal value for each node.

The calculation is performed backwards and starts at the stabilization point, which for Instrumental Meteorological Conditions (IMC) is defined 1000 feet above airport elevation with the aircraft in landing configuration, and finishes at the aircraft position. Nodes are generated according to discrete control values and the search space is pruned by means of problem constraints. Control variables are usually continuous functions discretized for generating next-nodes array based on a span distance defined by a set of events; for instance, an event occurs when a node matches or crosses an altitude constraint as in Fig. 2(a), or a speed limitation as displayed in Fig. 2(b). Additionally, any node dropping within the neighborhood limits of another is considered to be the same state, so if the existing node is in the open lists, path costs are compared and that with the lowest value is retained, while if the node is already in the closed list, the new node is discarded. This neighborhood zone is defined as the ellipsoid formed by distance, altitude and speed increments around the former node (gray-stripped node), which is illustrated in Fig. 2(c).

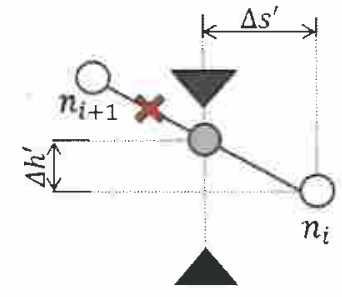

(a) Altitude constraint crossing.

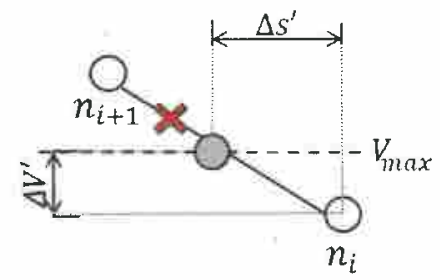

(b) Flight envelope matching.

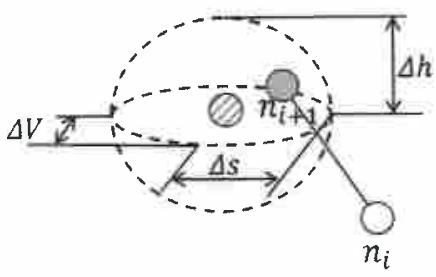

(c) Neighborhood zone around a node.

Figure 2. Examples of events related to the discretization of the control.

The resulting search space at the end of the computation is displayed in Fig. 3, which terminates when a. node falls within the neighborhood zone around the target and there is no more promising node in the Open list. From that node, parents are retrieved until the start node is attained, so the projections on the planes form the altitude and speed profile. The constraints (magenta triangles) prune the search space as the trajectory complies with the procedure design. From an operational point of view, flight crew would follow the computed path manually or automatically except when any circumstance forces the aircraft to deviate from the intended route. In that case, the calculation shall be relaunched to adapt the flight strategy to the dynamic aircraft state (tactical approach).

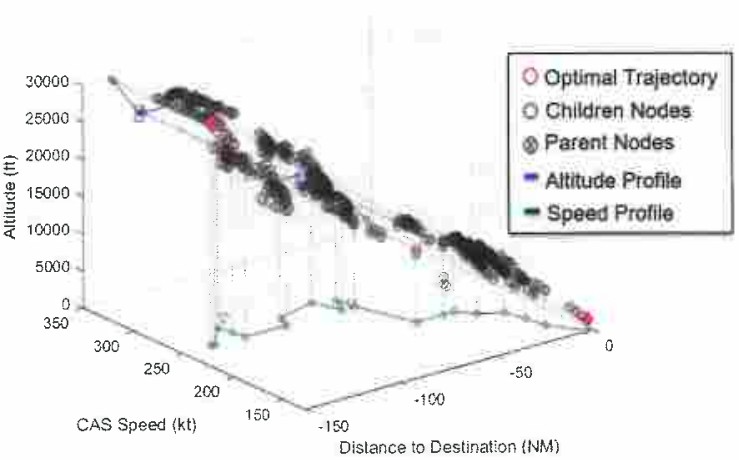

Figure 3. Search Space graph. In case that a solution does not exist due to the procedure design and aircraft performance, the algorithm explores all candidate nodes and produces an error message, which generally takes large computation times. 


\section{Results and Findings}

This chapter presents a series of use cases selected that permit to analyze the generated trajectories. Results are compared with those of a standalone Airbus FMS simulator hosted in a computer, which is representative enough for vertical profile comparison purposes. The optimization variable for this study is the fuel consumption. Three use cases are presented: $\mathrm{A}$ and $\mathrm{B}$ optimize the arrival path and $\mathrm{C}$ solves an aircraft over-energy scenario. The general parameters of the simulation used for the generation of the optimal trajectory are gathered in table 1.

Table 1. General simulation parameters for the case studies.

\begin{tabular}{cc}
\multicolumn{2}{c}{ Parameter } \\
\hline Aircraft type & $\mathrm{A} 320$ \\
Cost Index, $\mathrm{kg} / \mathrm{min}$ & 0 \\
Gross weight, $\mathrm{t}$ & 60 \\
Wind, $\mathrm{m} / \mathrm{s}$ & 0
\end{tabular}

\section{A. Profile Optimization at LFBO airport}

The first use case corresponds to a Required Navigation (RNAV) procedure at Toulouse airport (LFBO) based on an Instrument Landing System (ILS) approach to runway 32L whose parameters of the simulation are displayed in table 2. The initial entry point is NARAK waypoint (NARA6A procedure).

Table 2. Aircraft initial and final state at LFBO airport.

\begin{tabular}{ccc} 
& Initial state & Final state \\
\hline Distance to destination, NM & -3.14 & -108 \\
Altitude, ft & 1500 & 30000 \\
Speed, kt & 129.1 & 250 \\
Flap settings & full & clean
\end{tabular}

This procedure contains four altitude constraints (one AT OR BELOW $17000 \mathrm{ft}$ and three AT OR ABOVE $8000 \mathrm{ft}, 4000 \mathrm{ft}$ and $4000 \mathrm{ft}$ ). A vertical discontinuity occurs at $-25 \mathrm{NM}$; the aircraft does not dissipate enough energy with half airbrakes extended in order to decelerate and follow the required fight path. On the contrary, $A^{*}$ algorithm solves the discontinuity due to a speed reduction at that point and assuming full airbrakes extension (Fig. 4(b)) to increase the energy rate of change. Apart from solving the vertical discontinuity, fuel savings of $6 \%$ are obtained due to the removal of the geometric segment constructed by the FMS between -35 NM and -72 NM. The algorithm proposes a level flight at 270 kt then a smooth deceleration with a -2.8 flight path angle. In general, it is beneficial to add more thrust for a short level-off instead of less amount of thrust for a long geometric segment. Descent time is reduced by $3 \%$ as a result of the speed increase during the level flight segment and the fact that true airspeed is bigger for a higher altitude below the crossover altitude.

The vertical trajectory computed by the $\mathrm{A}^{*}$ algorithm can be coupled with a lateral path and terrain data $^{2}$ in order to define the complete arrival procedure as depicted in Fig. 5. The distance to destination from aircraft position is given and can be based on the lateral path defined by the procedure but also on the direct distance to the Final Approach Fix (FAF). Note that the bank angle is neglected in the computation of the vertical profile but aircraft track angle is used for projecting the wind component on the aircraft axis.

\section{B. Profile Optimization at KLAX airport}

The second use case finds an optimal path for the CDO procedure (SEAVU2) corresponding to an ILS approach to runway 24L at Los Angeles airport (KLAX) with entry point at SEAVU waypoint, table 3 defines the initial and final states. This procedure contains one speed constraint (AT OR BELOW $270 \mathrm{kt}$ ) 

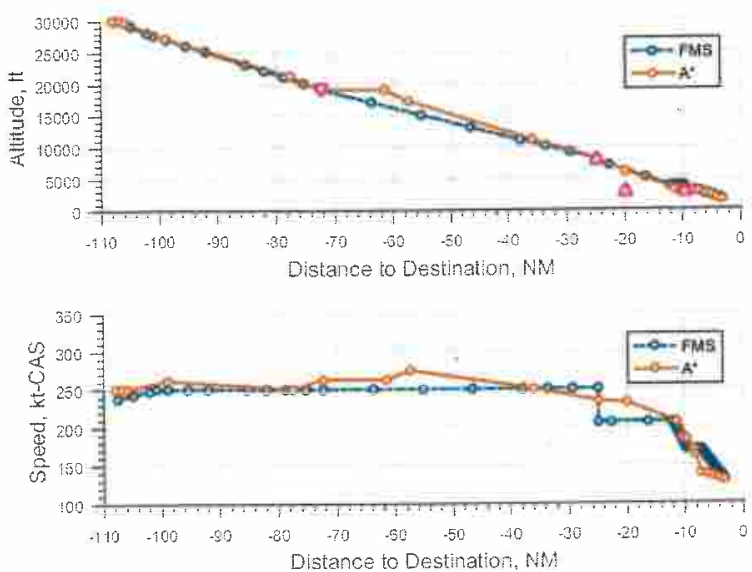

(a) Altitude (upper) and speed (bottom) profiles.
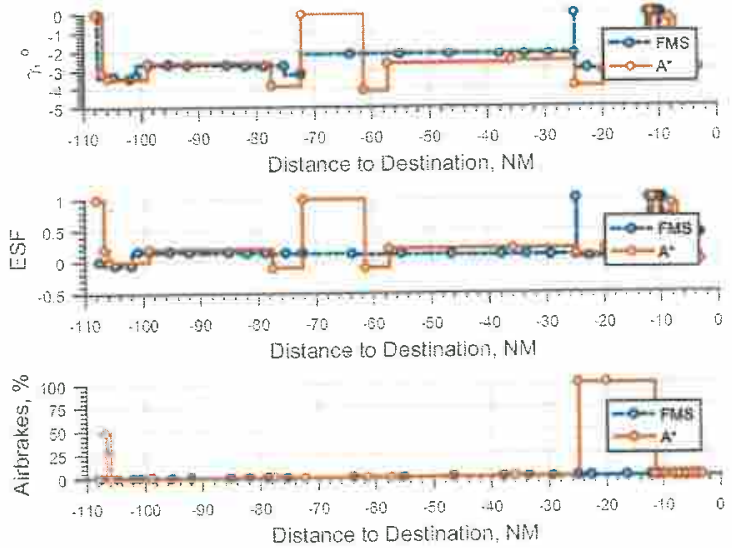

(b) Flight path angle (upper), Energy Share Factor (middle) and airbrakes (bottom).

Figure 4. LFBO use case: Comparison of trajectory generated by $A^{*}$ algorithm and FMS simulator.

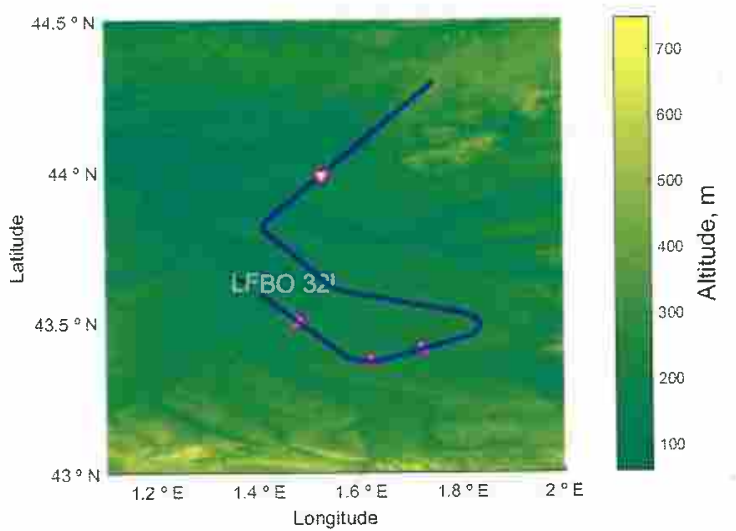

(a) Lateral path.

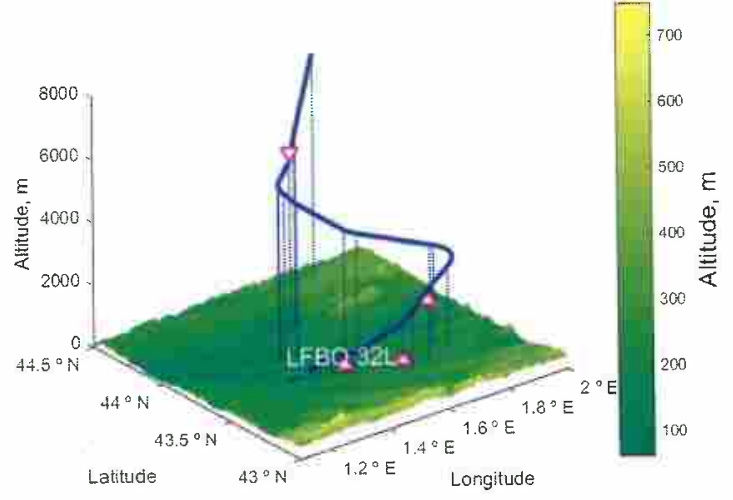

(b) Full arrival trajectory.

Figure 5. Vertical trajectory coupled to the lateral path defined by LFBO RNAV ILS-32L procedure.

and several altitude constraints (one AT $17000 \mathrm{ft}$, one WINDOW between 14000 - 12000 and ten AT OR ABOVE $16000,14000,10000,9000,8000,7000,6000,5000,4000$ and 4000 feet) that complicate the aircraft deceleration towards the approach speed.

Table 3. Aircraft initial and final state at KLAX airport.

\begin{tabular}{ccc} 
& Initial state & Final state \\
\hline Distance to destination, NM & -3.14 & -108 \\
Altitude, ft & 1125 & 30000 \\
Speed, kt & 129.1 & 250 \\
Flap settings & full & clean
\end{tabular}

Cruise phase is stretched so the aircraft flies higher and descends at higher rate in order to increase the speed, which is then reduced to satisfy the altitude constraints, which explains the kinetic peak occurring at -63 NM in Fig. 6(a). The main fuel savings in this procedure are obtained during the two-part geometric 

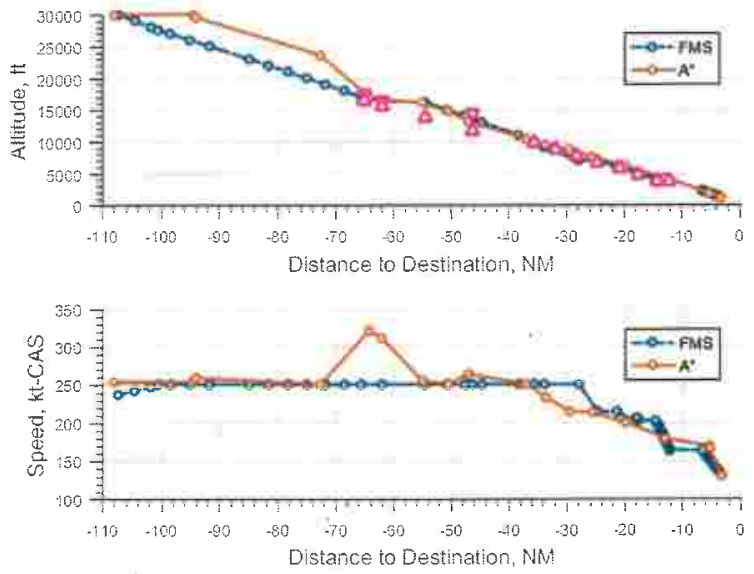

(a) Altitude (upper) and speed (bottom) profiles.
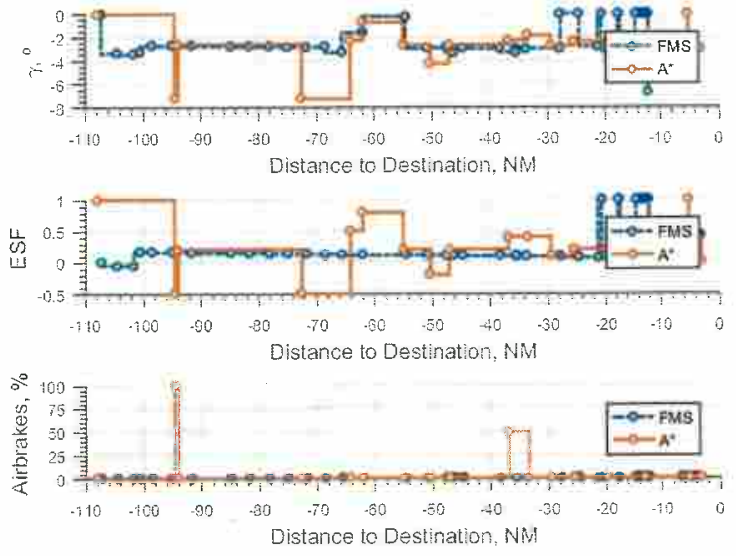

(b) Flight path angle (upper), Energy Share Factor (middle) and airbrakes (bottom)

Figure 6. KLAX use case: comparison of trajectory generated by $\mathrm{A}^{*}$ algorithm and FMS simulator.

segment that occurs from -25 NM to $-45 \mathrm{NM}$ and from $-55 \mathrm{NM}$ to $-65 \mathrm{NM}$, and during the constant speed segment in approach due to the slow deceleration for that aerodynamic configuration in a slope of -3 degrees. The algorithm produces a shorter time descent $(-3 \%)$ and reduces fuel consumption by a $12 \%$, since for the same speed adding thrust at cruise level is cheaper than at any other altitude. A short use of airbrakes is performed at -94 NM probably coming from a discretization error; additional constraints could forbid this kind of operational behavior in order to facilitate the flyability of the trajectory and avoid excessive pilots actions in manual modes.

\section{High-energy in Approach}

In this use case, the aircraft is located close from runway threshold and relatively high and fast to identify the high-energy situation, since flight crew is not able to stabilize aircraft state without additional drag. The initial and final aircraft state is described in table 4.

Table 4. High-energy use case simulation parameters.

\begin{tabular}{ccc} 
& Initial state & Final state \\
\hline Distance to destination, NM & -3.14 & -19.5 \\
Altitude, ft & 1500 & 7500 \\
Speed, kt & 129.5 & 250 \\
Flap settings & full & clean
\end{tabular}

In this situation, pilots usually apply a set of rules of thumb to estimate the best strategy to dissipate the energy excess and act consequently on the control devices. However, there are several flight strategies to solve the over-energy. On one hand, in the strategy 1, illustrated in Fig. 7(a), flight crew anticipates aerodynamic changes and extends airbrakes only if necessary. On the other hand, Fig. 7(b) shows strategy 2 where pilots rely on airbrakes extension as the main source to dissipate the energy and flap changes are retarded as much as possible. The impact on fuel consumption is that strategy 1 consumes $18 \%$ more fuel than strategy 2 due to the fact that idle settings change with the aerodynamic configuration in order to comply with certification, but reduces airbrakes solicitation by a $55 \%$. Furthermore strategy 1 provides an additional safety margin, since airbrakes could still being deployed in case of an unexpected tailwind while strategy 2 would require to initiate the go-around. As a consequence, the design of the function should privilege the flap anticipation and compensate any unexpected wind with airbrakes, which represents a margin for pilots. In general, a go-around procedure as a result of an unstabilized approach penalizes more than the fact of 

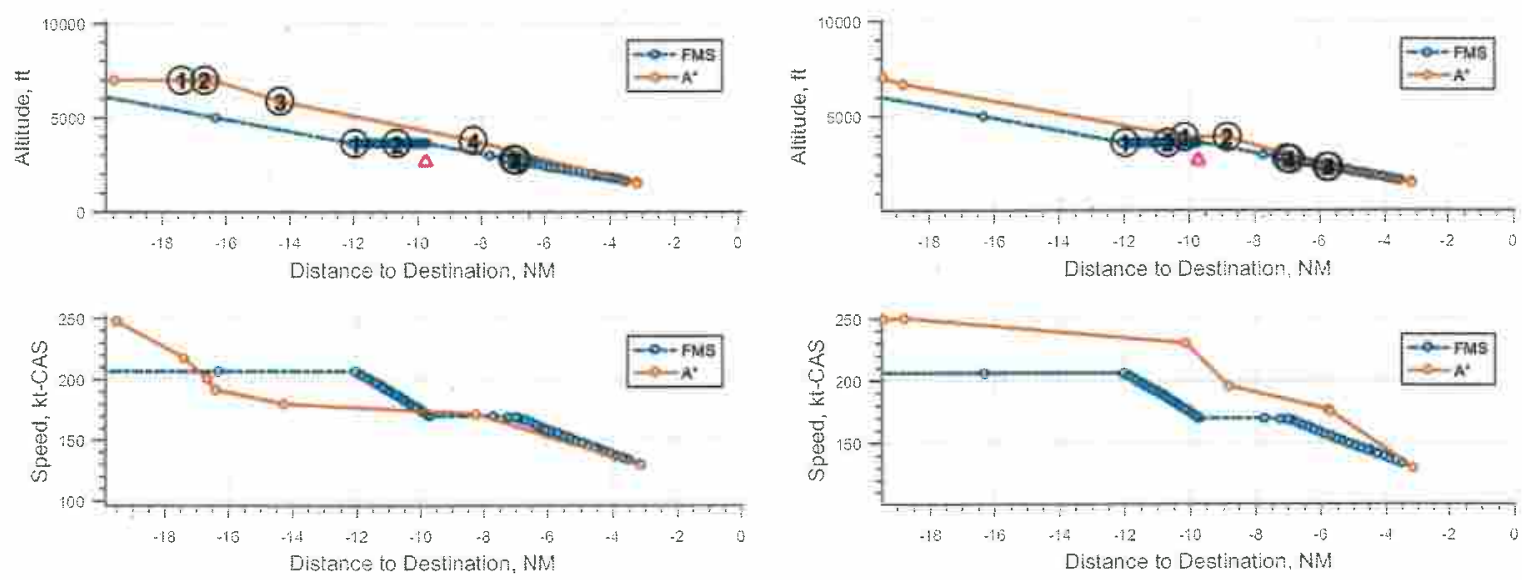

(a) Strategy 1: Flap-change anticipation with complementary airbrakes extension.

(b) Strategy 2: Airbrakes extension with retarded flap-change.

Figure 7. High-energy function design based on two different energy management strategies.

increasing fuel consumption during the final approach. A level-off during a high-energy approach may not be an obvious maneuver for a pilot that aims to stabilize the aircraft before landing but is an efficient strategy as it decelerates better and landing (aerodynamic) configuration generates more drag to dissipate any excess energy.

Note that the blue-dashed FMS trajectory in Figs. 7 and 8 only represents the nominal trajectory constructed by the FMS for this procedure and highlights the high-energy situation.
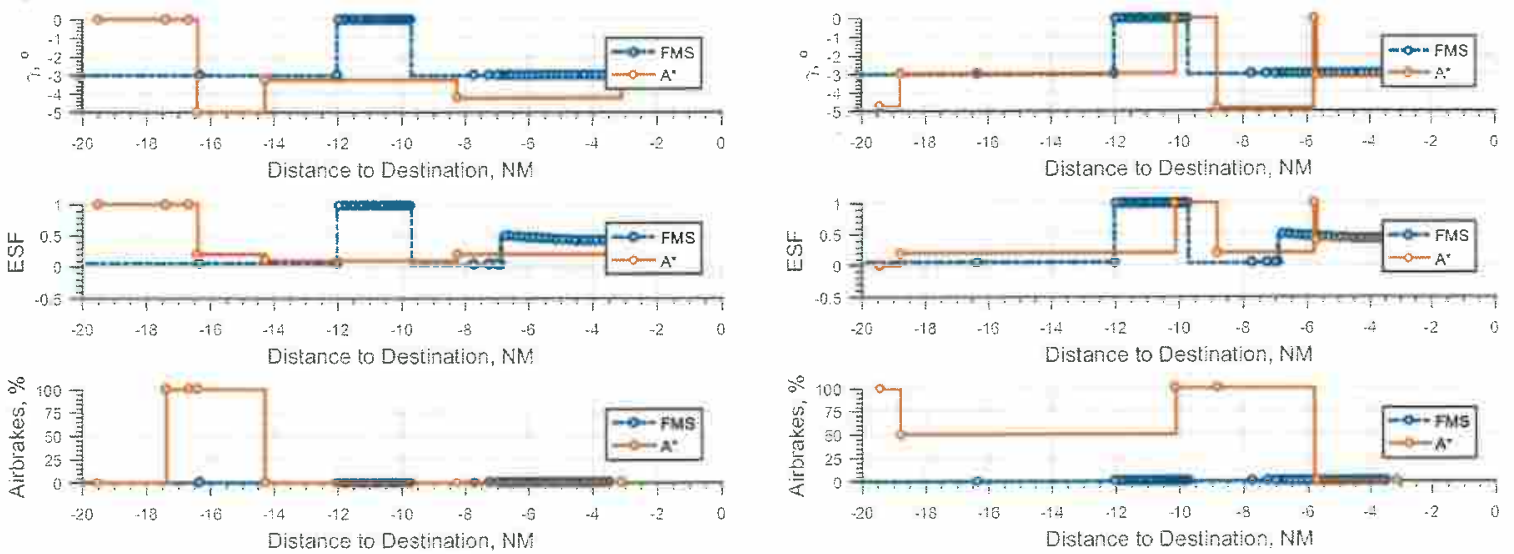

(a) Strategy 1: Flight path angle (upper), Energy Share Factor (middle) and airbrakes (bottom).

(b) Strategy 2: Flight path angle (upper), Energy Share Factor (middle) and airbrakes (bottom).

Figure 8. Control variables for the high-energy scenario.

\section{Conclusion}

This paper proposes an algorithm that generates upstream optimal trajectories with the certainty of reaching aircraft position. The computation is dynamically computed depending on aircraft state and solves any over-energy occurring during the approach phase. The algorithm is designed to solve any type of arrival 
procedure although the paper presents only three use cases; the first two show fuel savings of $6 \%$ and $12 \%$ with respect to a FMS simulator due to the fact that aircraft trades kinetic and potential energy and satisfies the whole set of constraints. The last use case highlights the fact that in high-energy situations during approach, safety is more important than optimization. In this case, two flight strategies lead to the same final state but the best trajectory is that which provides a margin to dissipate any unexpected energy error by means of airbrakes, therefore flap extensions are anticipated despite penalizing fuel consumption. Additionally, the study demonstrates that profiles based on enhanced energy management solve most of the vertical discontinuities with respect to current FMS design. In conclusion, the main contribution of the paper is to provide an algorithm that computes fuel-optimal trajectories that reach aircraft position (continuity of the trajectory) regardless to its energy-state and whose design solves high-energy situations during approach through flap and airbrakes adjustments.

As for the future perspectives, the main improvement axis is to work on a meaningful heuristic function that provides better estimations so that computation times are reduced. Furthermore, the operational concept could be tested in a simulated environment in order to prove the feasibility of the computed trajectories and compare the theoretic savings with respect to a real version of FMS software. Another future improvement could be the introduction of energy-limit strategies to warn pilots in the absence of solution and propose a stretched lateral path to dissipate the excess energy, so the algorithm antomatically knows a priori if a solution exists without the need of launching the whole calculation.

\section{References}

${ }^{1}$ Airbus, "Global Market Forecast 2016-2035 - Mapping Demand," 2016

${ }^{2}$ Eurocontrol, "European Aviation in 2040. Challenges of Growth," Annex 4, Network Congestion, 2018.

${ }^{3}$ Eurocontrol, "All-Causes Delay to Air Transport in Europe," March 2017.

${ }^{4}$ EASA, Continued Growth of Aviation poses Environmental Challenges, European Aviation Safety Agency, 2019

${ }^{5}$ Eurocontrol, Free Route Airspace developments, European Organisation for the Safety of Air Navigation, December 2016.

${ }^{6} \mathrm{ICAO}$, Continuous Descent Operations (CDO) Manual - Doc 9931 AN/476, International Civil Aviation Organization, Montreal, Canada, 2010.

${ }^{7}$ Fricke, H., Seis, C., and Herrmann, R., "Fuel and Energy Benchmark Analysis of Continuous Descent Operations," Air Traffuc Control Quarterly, Vol. 23(1) pp. 83108 (2015), July 2015.

${ }^{8}$ Liden, S., "The evolution of Flight Management Systems," Honeywell Air Tromport Systems, PO Box, Vol. 21, No. 11, 1994, pp. 7803-24254.

${ }^{9}$ Walter, R., "Flight Management Systems," 2001.

${ }^{10}$ Blajev, T. and Curtis, C. W., Go-Around Decision-Making and Execution Project, Flight Safety Foundation, March 2017.

${ }^{11}$ Betts, J. T. and Cramer, E. J., "Application of direct transcription to commercial aircraft trajectory optimization," Journal of Guidance, Control and Dynamics, Vol. 18, No. 1, 1995, pp. 151-159.

${ }^{12}$ Delahaye, D., Puechmorel, S., Tsiotras, P., and Feron, E., "Mathematical Models for Aircraft Trajectory Design : A Survey," Lecture notes in Electrical Engineering Lecture Notes in Electrical Engineering, Vol. 10, No. 1007, 2014, pp. 43154475 .

${ }^{13}$ De Jong, P. M. A., De Gelder, N., Bussink, F. J. L., Verhoeven, R. P. M., Kohrs, R., and Mulder, M., "Time and Energy Management during Descent and Approach for Aircraft: A Batch-Simulation Study," Journal of Aircraft, 2013.

${ }^{14}$ Fernandes, R. and Beskens, C., "Benefits of Optimal Flight Planning on Noise and Emissions Abatement at the Frankfurt Airport," EADS Innovation Works. University of Bremen, Bremen, Germany, Vol. 2

${ }^{15}$ Park, S. G. and Clarke, J. P., "Vertical Trajectory Optimization for Continuous Descent Arrival Procedure," AIAA Guidance, Navigation, and Control Conference, Minneapolis, Minnesota, August 2012.

${ }^{16}$ Park, S. G. and Clarke, J. P., "Vertical Trajectory Optimization to Minimize Environmental Impact in the Presence of Wind," August 2015.

${ }^{17}$ Devulapalli, R., "An Efficient Algorithm for Commercial Aircraft Trajectory Optimization in the Air Traffic System," .

${ }^{18}$ E. Rippel, A. B.-G. and Shimkin, N., "Fast Graph-Search Algorithms for General Aviation Flight Trajectory Generation," TechnionIsrael Institute of Technology, Haifa, Israel.

${ }^{19}$ J. Doebbler, P. Gesting, J. V., "Real-time Path Planning and Terrain Obstacle Avoidance for General Aviation Aircraft," AIAA Guidance, Navigation, and Control Conference and Exhibit. AIAA 2005-5825.

${ }^{20}$ Peter E. Hart, Nils J.Nilsson, B. R., "A formal Basis for the Heuristic Determination of Minimum Cost Paths," IEEE Transactions of systems science and cybernetics, Vol.SSC-4,NO.2.

${ }^{21}$ L.Schultz, R. and R.Zagalsky, N., "Aircraft Performance Optimization," Journal of Aircraft, 1972.

${ }^{22}$ ARINC, "Navigation System Database," Aeronautical Radio Incorporated, ARINC Specification 424-22, July 2018.

${ }^{23}$ Andreu-Altava, R., Mere, J., Delahaye, D., and Miquel, T., "Flight Management System Pathfinding Algorithm for Automatic Trajectory Generation," 37th Digital Avionics System Conference, London, UK, September 2018.

${ }^{24}$ A., J., Reuter, H., Nelson, A., and Guevara, E., "Hole-filled seamless SRTM data V4, International Centre for Tropical Agriculture (CIAT)," http://srtm.csi.cgiar.org, 2008. 
